\title{
Leading neutron production at the EIC and LHeC: Estimating the impact of the absorptive corrections
}

\author{
F. Carvalho, ${ }^{1}$ V. P. Gonçalves, ${ }^{2}$ F. S. Navarra, ${ }^{3}$ and D. Spiering ${ }^{3}$ \\ ${ }^{1}$ Departamento de Ciências Exatas e da Terra, Universidade Federal de São Paulo, Campus Diadema, \\ Rua Prof. Artur Riedel, 275, Jd. Eldorado, 09972-270, Diadema, SP, Brazil \\ ${ }^{2}$ High and Medium Energy Group, Instituto de Física e Matemática, \\ Universidade Federal de Pelotas Caixa Postal 354, 96010-900, Pelotas, RS, Brazil \\ ${ }^{3}$ Instituto de Física, Universidade de São Paulo, C.P. 66318, 05315-970 São Paulo, SP, Brazil
}

(Received 24 November 2020; accepted 4 February 2021; published 25 February 2021)

\begin{abstract}
Leading neutron production in $e p$ collisions at high energies is investigated using the color dipole formalism and taking into account saturation effects. We update the treatment of absorptive effects and estimate the impact of these effects on leading neutron spectra in the kinematical range that will be probed by the Electron Ion Collider (EIC) and by the Large Hadron electron Collider (LHeC). We demonstrate that Feynman scaling, associated with saturation, is not violated by the inclusion of absorptive effects. Moreover, our results indicate that the leading neutron spectrum is strongly suppressed at small photon virtualities. These results suggest that absorptive effects cannot be disregarded in future measurements of the $\gamma \pi$ cross section to be extracted from data on leading neutron production.
\end{abstract}

DOI: 10.1103/PhysRevD.103.034021

\section{INTRODUCTION}

The study of deep inelastic electron-proton $(e p)$ scattering has significantly improved our understanding of the proton structure in the high energy (small- $x$ )regime (For a recent review see, e.g., Ref. [1]). In the future the partonic structure of other hadrons will be investigated [2]. The pion structure has been discussed by several authors [3-9] and the subject became recently a hot topic due to the prospect of measuring the pion structure function $F_{2}^{\pi}\left(x, Q^{2}\right)$ in future electron-hadron colliders at the BNL and CERN [10,11]. The basic idea is that the pion structure can be probed in electron-proton collisions through the Sullivan process [12], where the electron scatters off the meson cloud of the proton target. The associated processes can be separated by tagging a forward neutron in the final state, which carries a large fraction of the proton energy. Theoretically, this leading neutron production is usually described assuming that the splitting $p \rightarrow \pi^{+} n$ and the photon-pion interaction can be factorized, as represented in Fig. 1(a), where $f_{\pi / p}$ represents the pion flux. Assuming the validity of the factorization hypothesis and the universality of the fragmentation process, which allows us to constrain $f_{\pi / p}$ using the data of leading neutron production in

Published by the American Physical Society under the terms of the Creative Commons Attribution 4.0 International license. Further distribution of this work must maintain attribution to the author(s) and the published article's title, journal citation, and DOI. Funded by SCOAP ${ }^{3}$. pp collisions, we can obtain $\sigma^{\gamma^{*} \pi}$ and, consequently, determine the $x$ and $Q^{2}$ dependencies of the pion structure function. However, the validity of this procedure is limited by absorptive effects, denoted by $S_{e i k}^{2}$ in Fig. 1, that are associated with soft rescatterings between the produced and spectator particles. The studies performed in Refs. [13-15] indicated that these effects strongly affect leading neutron production in $p p$ collisions. In contrast, the absorptive corrections are predicted to be smaller in $e p$ collisions and their effects become weaker at larger photon virtualities $[6,8,13,16-18]$. Although the treatment of the absorptive corrections has advanced in recent years, they are still one of the main uncertainties in the study of leading neutron production in $p p$ collisions at RHIC and LHC and $e p$ collisions at the EIC and $\mathrm{LHeC}$.

In Refs. $[19,20]$ we proposed a model to treat leading neutron production in ep processes based on the color dipole formalism [21]. In this model, the virtual photonpion cross section can be factorized in terms of the photon wave function (which describes the photon splitting into a $q \bar{q}$ pair) and the dipole-pion cross section $\sigma_{d \pi}$, as represented in Fig. 1(b). As shown in Refs. [19,20], the HERA data are quite well described by this approach assuming that absorptive corrections can be factorized and represented by a multiplicative constant factor, denoted by $K$ in Ref. [19]. Although successful (in the limited kinematical range probed by HERA) and a reasonable assumption to obtain a first approximation of the cross sections for the EIC and $\mathrm{LHeC}$, it is fundamental to improve the description of $S_{e i k}^{2}$ in order to derive more realistic predictions. 


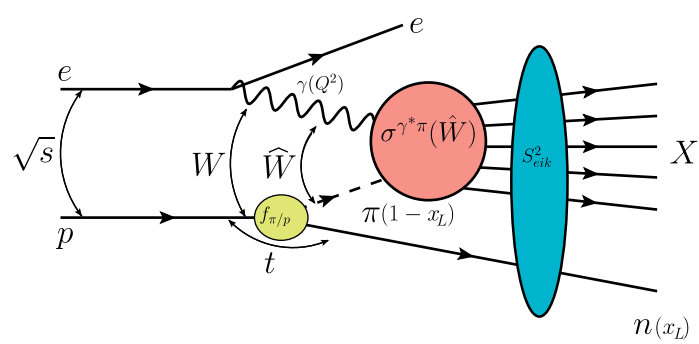

(a)

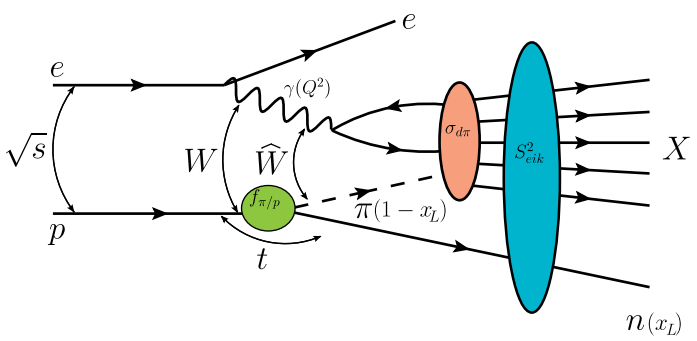

(b)

FIG. 1. (a) Leading neutron $n$ production in $e p \rightarrow e n X$ interactions at high energies. (b) Description of the process in the color dipole model.

Our goal in this paper is to revisit and update the approach proposed in Ref. [13] for the absorptive effects. This approach allows us to estimate these effects in terms of the color dipole formalism, i.e., using the same ingredients of the model proposed in $[19,20]$. As a consequence, we will be able to derive parameter free predictions for the cross sections, which can be directly compared with the HERA data. Moreover, we will estimate the strength of the absorptive effects for different photon virtualities and center-of-mass energies and present predictions for leading neutron production in future colliders.

\section{FORMALISM}

Initially, let us discuss the approach proposed in Ref. [19] to treat the leading neutron production in $e p$ collisions, disregarding the absorptive effects. At high center-of-mass energies, this process can be seen as a set of three factorizable subprocesses [see Fig. 1(b)]: (i) the photon emitted by the electron fluctuates into a quarkantiquark pair (the color dipole), (ii) the color dipole interacts with the pion, and (iii) the leading neutron is formed. In the color dipole formalism, the differential cross section reads:

$$
\begin{aligned}
& \frac{d^{2} \sigma\left(W, Q^{2}, x_{L}, t\right)}{d x_{L} d t}=f_{\pi / p}\left(x_{L}, t\right) \sigma_{\gamma^{*} \pi}\left(\hat{W}^{2}, Q^{2}\right), \\
& =f_{\pi / p}\left(x_{L}, t\right) \\
& \quad \times \int_{0}^{1} d z \int d^{2} r \sum_{L, T}\left|\Psi_{T, L}\left(z, r, Q^{2}\right)\right|^{2} \sigma_{d \pi}\left(x_{\pi}, r\right)
\end{aligned}
$$

where $Q^{2}$ is the virtuality of the exchanged photon, $x_{L}$ is the proton momentum fraction carried by the neutron, and $t$ is the square of the four-momentum of the exchanged pion. Moreover, $\hat{W}$ is the center-of-mass energy of the virtual photon-pion system, which can be written as $\hat{W}^{2}=\left(1-x_{L}\right) W^{2}$, where $W$ is the center-of-mass energy of the virtual photon-proton system. In terms of the measured quantities $x_{L}$ and transverse momentum $p_{T}$ of the neutron, the pion virtuality is

$$
t \simeq-\frac{p_{T}^{2}}{x_{L}}-\frac{\left(1-x_{L}\right)\left(m_{n}^{2}-m_{p}^{2} x_{L}\right)}{x_{L}}
$$

In Eq. (2), the virtual photon-pion cross section was expressed in terms of the transverse and longitudinal photon wave functions $\Psi_{i}$, which describe the photon splitting into a $q \bar{q}$ pair of size $r \equiv|r|$, and the dipole-pion cross section $\sigma_{d \pi}$, which is determined by the QCD dynamics at high energies [22]. The variable $z$ represents the longitudinal photon momentum fraction carried by the quark, the variable $r$ defines the relative transverse separation of the pair (dipole), and the scaling variable $x_{\pi}$ is defined by $x_{\pi}=x /\left(1-x_{L}\right)$, where $x$ is the Bjorken variable.

The flux factor $f_{\pi / p}$ gives the probability of the splitting of a proton into a pion-neutron system and can be expressed as follows (see e.g., Ref. [13]):

$$
f_{\pi / p}\left(x_{L}, t\right)=\frac{2}{3} \pi \sum_{\lambda \lambda^{\prime}}\left|\phi_{n \pi}^{\lambda \lambda^{\prime}}\left(x_{L}, \mathbf{p}_{T}\right)\right|^{2}
$$

where $\phi_{n \pi}^{\lambda \lambda^{\prime}}\left(x_{L}, \mathbf{p}_{T}\right)$ is the probability amplitude to find, inside a proton with spin up, a neutron with longitudinal momentum fraction $x_{L}$, transverse momentum $\mathbf{p}_{T}$, and helicity $\lambda$ and a pion, with longitudinal momentum fraction $1-x_{L}$, transverse momentum $-\mathbf{p}_{T}$, and helicity $\lambda^{\prime}$. In the light-cone approach, the amplitudes $\phi_{n \pi}$ of a proton with spin $+1 / 2$, read

$$
\begin{aligned}
\phi_{n \pi}^{1 / 2,0}\left(x_{L}, \mathbf{p}_{T}\right) & =\frac{\sqrt{3} g_{0}}{4 \pi \sqrt{\pi}} \frac{1}{\sqrt{x_{L}^{2}\left(1-x_{L}\right)}} \frac{m_{n}\left(x_{L}-1\right)}{M_{n \pi}^{2}-m_{n}^{2}}, \\
\phi_{n \pi}^{-1 / 2,0}\left(x_{L}, \mathbf{p}_{T}\right) & =\frac{\sqrt{3} g_{0}}{4 \pi \sqrt{\pi}} \frac{1}{\sqrt{x_{L}^{2}\left(1-x_{L}\right)}} \frac{\left|\mathbf{p}_{T}\right| e^{-i \varphi}}{M_{n \pi}^{2}-m_{n}^{2}},
\end{aligned}
$$

where $M_{n \pi}^{2}$ is the invariant mass of the pion-neutron system, given by

$$
M_{n \pi}^{2}=\frac{m_{n}^{2}+p_{T}^{2}}{x_{L}}+\frac{m_{\pi}^{2}+p_{T}^{2}}{1-x_{L}},
$$

with $m_{n}$ and $m_{\pi}$ being the neutron and the pion masses, $g_{0}$ is the bare pion-nucleon coupling constant, and $\varphi$ is the 
azimuthal angle in the transverse plane. Because of the extended nature of the hadrons involved, the interaction amplitudes in the above equations have to be modified by including a phenomenological $\pi N N$ form factor, $G\left(x_{L}, p_{T}\right)$. It is important to stress here that while the vertex is derived from an effective meson-nucleon Lagrangian, the form factor is introduced ad hoc. In our analysis we will choose the covariant form factor, corrected by the Regge factor, given by

$$
G\left(x_{L}, p_{T}\right)=\exp \left[R_{c}^{2}\left(t-m_{\pi}^{2}\right)\right]\left(1-x_{L}\right)^{-t}
$$

where $R_{c}^{2}=0.3 \mathrm{GeV}^{2}$ was constrained using the HERA data (for details see Ref. [19]). The amplitude $\phi_{n \pi}^{\lambda \lambda^{\prime}}\left(x_{L}, \mathbf{p}_{T}\right)$ changes to $\phi_{n \pi}^{\lambda \lambda^{\prime}}\left(x_{L}, \mathbf{p}_{T}\right) G\left(x_{L}, p_{T}\right)$ and then the pion flux becomes

$$
f_{\pi / p}\left(x_{L}, t\right)=\frac{2}{3} \pi \sum_{\lambda \lambda^{\prime}}\left|\phi_{n \pi}^{\lambda \lambda^{\prime}}\left(x_{L}, \mathbf{p}_{T}\right)\right|^{2}\left|G\left(x_{L}, p_{T}\right)\right|^{2},
$$

where $2 / 3$ is the isospin factor and the azimuthal angle in the transverse plane has been integrated out.

In order to include the absorptive effects in our predictions for the leading neutron spectrum $d \sigma / d x_{L}$, we will follow the approach proposed in Ref. [13], where these effects were estimated using the high-energy Glauber approximation [23] to treat the multiple scatterings between the dipole and the pion-neutron system. As demonstrated in Ref. [13], such approach can be easily implemented in impact parameter space, implying that the spectrum can be expressed as follows:

$$
\begin{aligned}
\frac{d \sigma\left(W, Q^{2}, x_{L}\right)}{d x_{L}}= & \int d^{2} b_{\text {rel }} \rho_{n \pi}\left(x_{L}, b_{\text {rel }}\right) \\
& \times \int d z d^{2} r \sum_{L, T}\left|\Psi_{T, L}\left(z, r, Q^{2}\right)\right|^{2} \\
& \times \sigma_{d \pi}\left(x_{\pi}, r\right) S_{e i k}^{2}\left(r, b_{\text {rel }}\right),
\end{aligned}
$$

where $\rho_{n \pi}\left(x_{L}, b_{\text {rel }}\right)$ is the probability density of finding a neutron and a pion with momenta $x_{L}$ and $1-x_{L}$, respectively, and with a relative transverse separation $b_{\text {rel }}$, which is given by

$$
\rho_{n \pi}\left(x_{L}, b_{\text {rel }}\right)=\sum_{\lambda \lambda^{\prime}}\left|\psi_{n \pi}^{\lambda \lambda^{\prime}}\left(x_{L}, b_{\text {rel }}\right)\right|^{2} .
$$

with

$$
\psi_{n \pi}^{\lambda \lambda^{\prime}}\left(x_{L}, b_{\mathrm{rel}}\right)=\frac{1}{2 \pi} \int d^{2} \mathbf{p}_{T} e^{i b_{\mathrm{rel}} \cdot \mathbf{p}_{T}} \Phi_{n \pi}^{\lambda \lambda^{\prime}}\left(x_{L}, \mathbf{p}_{T}\right),
$$

and $\Phi_{n \pi}^{\lambda \lambda^{\prime}}=\sqrt{2 / 3} \phi_{n \pi}^{\lambda \lambda^{\prime}} G\left(x_{L}, p_{T}\right)$. The main assumption of the model proposed in Ref. [13] is that the pion and the neutron are well separated in phase space, which implies that the interaction between them is negligible and that the absorptive corrections can be factorized from the dipolepion interaction and estimated by taking into account the dipole-neutron scatterings. Such approximation is expected to be valid when the neutron has a large momentum fraction. However, the range of $x_{L}$ in which the model can be applied is still not well known and deserves more detailed studies in the future. Here we will follow Ref. [13] and we will assume that $S_{e i k}^{2}$ can be expressed in terms of the dipole-neutron $\left(\sigma_{d n}\right)$ cross section. Assuming that the scattering amplitude for the dipole-neutron scattering can be expressed by a Gaussian profile function [13], the survival factor $S_{e i k}^{2}$ associated with the absorptive effects will be given by

$S_{e i k}^{2}\left(r, b_{\text {rel }}\right)=\left\{1-\Lambda_{\text {eff }}^{2} \frac{\sigma_{d n}\left(x_{n}, r\right)}{2 \pi} \exp \left[-\frac{\Lambda_{\text {eff }}^{2} b_{\text {rel }}^{2}}{2}\right]\right\}$,

where $x_{n}=x / x_{L}$ and $\Lambda_{\text {eff }}^{2}$ is an effective parameter that was found to be equal to $0.1 \mathrm{GeV}^{2}$ in Ref. [13]. In our analysis, we will assume that $\sigma_{d n}$ is equal to the dipole-proton cross section, $\sigma_{d p}$, constrained by the HERA data. Finally, in order to estimate the spectrum, we must specify the dipolepion cross section, which is dependent on the description of the QCD dynamics at small $x$. As in Ref. [19], we will assume that this quantity can be related to the dipole-proton cross section using the additive quark model. Moreover, $\sigma_{d p}$ will be described by the color glass condensate (CGC) formalism, as given in the phenomenological model proposed in Ref. [24]. As a consequence, we will have that

$$
\begin{aligned}
\sigma_{d \pi}(x, r) & =\frac{2}{3} \cdot \sigma_{d p}(x, r) \\
& =\frac{2}{3} \cdot 2 \pi R_{p}^{2} \times \begin{cases}\mathcal{N}_{0}\left(\frac{r Q_{s}}{2}\right)^{2\left(\gamma_{s}+\frac{\ln \left(2 / r Q_{s}\right)}{K \lambda Y}\right)}, & \text { for } r Q_{s}(x) \leq 2, \\
1-\mathrm{e}^{-a \ln ^{2}\left(b r Q_{s}\right)}, & \text { for } r Q_{s}(x)>2,\end{cases}
\end{aligned}
$$

where $a$ and $b$ are determined by continuity conditions at $r Q_{s}(x)=2$. The parameters $\gamma_{s}=0.7376, \kappa=9.9$, $\mathcal{N}_{0}=0.7$, and $R_{p}=3.344 \mathrm{GeV}^{-1}$ have been adjusted using the HERA data in Ref. [25], with the saturation scale $Q_{s}$ being given by

$$
Q_{s}^{2}(x)=Q_{0}^{2}\left(\frac{x_{0}}{x}\right)^{\lambda}
$$

with $x_{0}=1.632 \times 10^{-5}, \lambda=0.2197, Q_{0}^{2}=1.0 \mathrm{GeV}^{2}$. The first line of Eq. (12) describes the linear regime whereas the second one includes saturation effects.

\section{RESULTS}

With the ingredients introduced in the previous section, we are ready to obtain parameter free predictions that can 


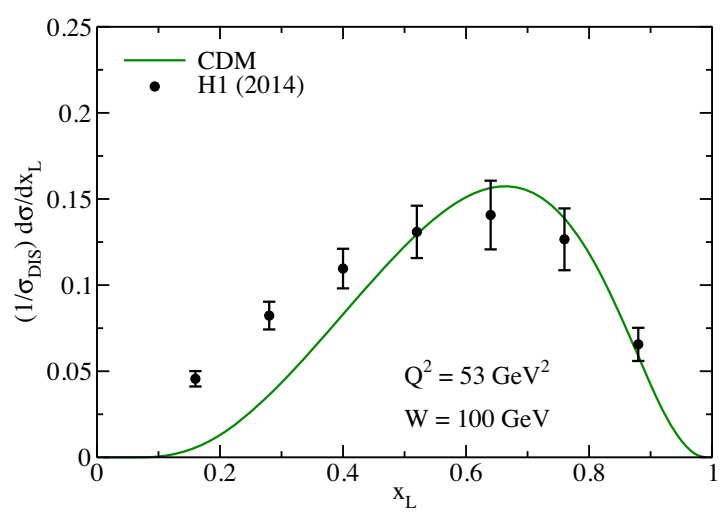

(a)

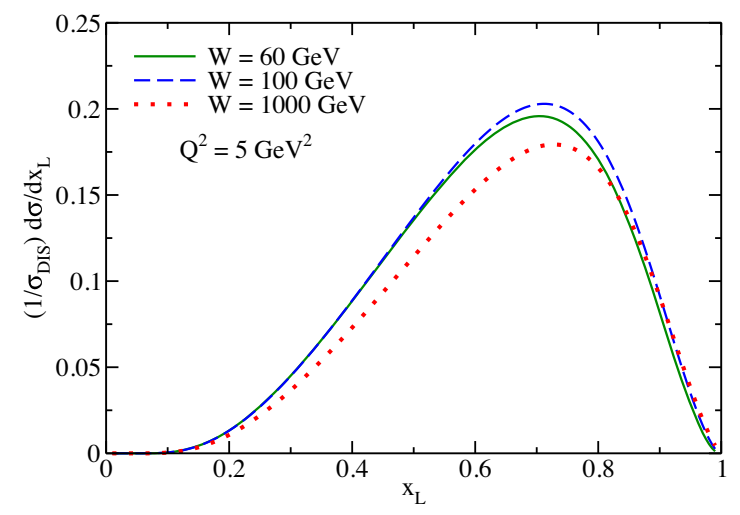

(b)

FIG. 2. (a) Comparison of the CDM prediction with the $\mathrm{H} 1$ data [26]. (b) Predictions for the spectra considering different center-ofmass energies and $Q^{2}=5 \mathrm{GeV}^{2}$.

be compared with the HERA data. We can also derive predictions which can be tested in future $e p$ colliders. In Fig. 2(a) the color dipole model (CDM) prediction for the kinematical range probed by HERA is presented. As it can be seen, the H1 data [26] are quite well described in the region $x_{L} \gtrsim 0.5$. As shown in previous studies [14,16], for smaller values of $x_{L}$, additional contributions are expected to play a significant role. We can estimate the leading neutron spectrum for a kinematical range beyond that probed by HERA. We are particularly interested in smaller values of the photon virtuality, where we expect a larger contribution of saturation effects, and for the center-ofmass energies that will be reached at the EIC and LHeC. The results are presented in Fig. 2(b). From the figure we see that the predictions are not strongly dependent on $W$. This is expected from the results presented in Ref. [19], where we have demonstrated that saturation leads to Feynman scaling, i.e., the energy independence of the $x_{L}$ spectra. Such scaling is expected to be strict when the saturation scale becomes larger than the photon virtuality, which is satisfied for small values of $Q^{2}\left(\lesssim 2 \mathrm{GeV}^{2}\right)$. However, as shown e.g., in Ref. [24], the presence of the saturation effects also modifies the behavior of the cross sections in a larger $Q^{2}$ range, implying the result observed in Fig. 2(b). In contrast, the Dokshitzer-Gribov-LipatovAltarelli-Parisi evolution leads to stronger violation of Feynman scaling, as shown in Ref. [19]. In a future experimental analysis of the leading neutron spectrum it will be very interesting to test this prediction of the color dipole model.

As discussed above, in order to measure the $\gamma \pi$ cross section and extract the pion structure function, it is crucial to have control of the absorptive effects in the kinematical range probed by the collider. In particular, we should know the dependence of these effects on $Q^{2}, W$, and $x_{L}$. We can estimate the impact of the absorptive effects through the calculation of the ratio between the cross sections with and without absorption, where the latter is estimated assuming $S_{e i k}^{2}=1$. Our predictions for this ratio, denoted $K_{a b s}$ hereafter, are presented in Fig. 3. Our results show that the impact increases for smaller values of $Q^{2}$ and larger energies $W$. For $Q^{2}=50 \mathrm{GeV}^{2}$, we see that $K_{a b s} \approx 0.9$ for $x_{L} \gtrsim 0.5$, with the predictions being similar for the three values of $W$. This weak absorption is expected in the color dipole model, since at large values of $Q^{2}$ the main contribution for the cross section comes from dipoles with a small pair separation. In this regime, denoted color transparency, the impact of the rescatterings is small, which

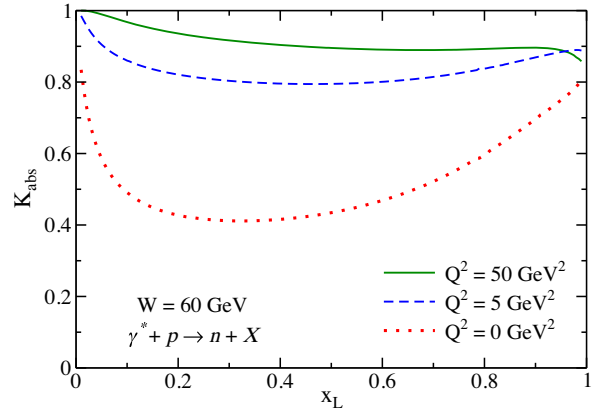

(a)

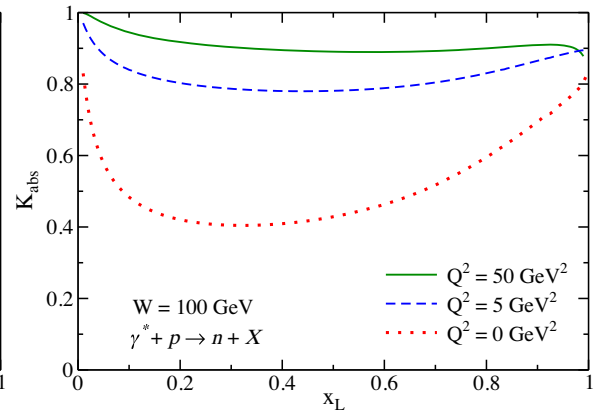

(b)

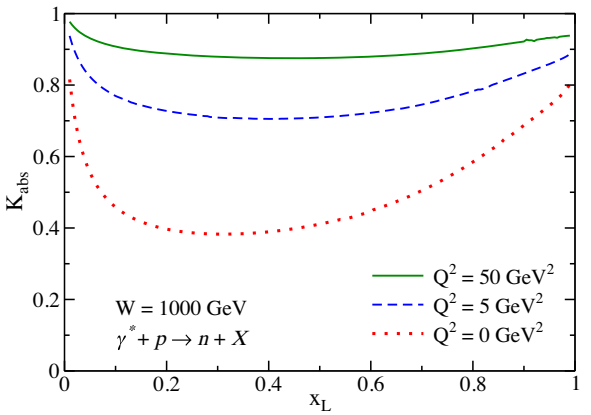

(b)

FIG. 3. Dependence of the absorptive effects on $x_{L}$ in leading neutron production in $e p$ collisions for different values of the photon virtuality (a) $W=60 \mathrm{GeV}$, (b) $W=100 \mathrm{GeV}$, and (c) $W=1000 \mathrm{GeV}$. 
implies that the absorptive effects become negligible. Another important aspect is that for large photon virtualities, the main effect of absorption is to suppress the cross section by a constant factor. Similar results were derived in Ref. [13]. On the other hand, for photoproduction $\left(Q^{2}=0\right)$, we observe strong absorptive effects, which reduce the cross sections by a factor $\approx 0.4$ for $x_{L}=0.5$. This result is also expected, since for small $Q^{2}$ the cross section is dominated by large dipoles and, consequently, the contribution of the rescatterings cannot be disregarded. For larger values of $x_{L}$, absorptive effects cannot be modeled by a constant factor. Our conclusions agree with those derived in Ref. [16] using Regge theory. Finally, our results indicate that the contribution of the absorptive effects is not strongly energy dependent. This result suggests that the main conclusion of Ref. [19], that the spectra will satisfy Feynman scaling, is still valid when the absorptive effects are estimated using a more realistic model, as already observed in Fig. 2(b).

\section{SUMMARY}

In this paper we have updated the treatment of the absorptive effects and incorporated them in the model proposed in our previous studies [19,20,27,28], which is based on the color dipole formalism. Using the approach proposed in Ref. [13], we have been able to derive parameter free predictions for the leading neutron spectra. We demonstrated that our model describes the HERA data in the region where the pion exchange is expected to dominate. Moreover, we have presented predictions for the kinematical ranges that will be probed by the future EIC and LHeC. Our results indicate that the leading neutron spectra are not strongly energy dependent at small photon virtualities. As shown in Ref. [19], this almost energy independence (Feynman scaling) is a consequence of saturation effects, which are expected to become significant at small $Q^{2}$ and large energies.

We have estimated the impact of the absorptive effects, demonstrated that they increase at smaller photon virtualities and that they depend on the longitudinal momentum $x_{L}$. Our results show that modeling these effects by a constant factor is a good approximation only for large $Q^{2}$. Our main conclusion is that a realistic measurement of the $\gamma \pi$ cross section in future colliders and the extraction of the pion structure function must take into account the important contribution of the absorptive effects, especially at small values of the photon virtuality, where these factorizationbreaking corrections pose a significant challenge to the program of trying to measure the parton distribution functions of the pion. As pointed out in our results, an alternative to determine these PDFs is to perform the analysis using the large- $Q^{2}$ data, where the absorptive corrections become negligible. Such results indicate that future experimental data on leading neutron production in $e p$ collisions at the EIC will be crucial to test the main assumptions of our model, as well as to improve our understanding of this important observable.

\section{ACKNOWLEDGMENTS}

This work was partially financed by the Brazilian funding agencies CNPq, FAPESP, FAPERGS, and INCT-FNA (process number 464898/2014-5).
[1] K. Kovařík, P. M. Nadolsky, and D. E. Soper, Rev. Mod. Phys. 92, 045003 (2020).

[2] A. C. Aguilar, Z. Ahmed, C. Aidala, S. Ali, V. Andrieux, J. Arrington, A. Bashir, V. Berdnikov, D. Binosi, L. Chang et al., Eur. Phys. J. A 55, 190 (2019).

[3] H. Holtmann, G. Levman, N. N. Nikolaev, A. Szczurek, and J. Speth, Phys. Lett. B 338, 363 (1994).

[4] B. Kopeliovich, B. Povh, and I. Potashnikova, Z. Phys. C 73, 125 (1996).

[5] M. Przybycien, A. Szczurek, and G. Ingelman, Z. Phys. C 74, 509 (1997).

[6] N. N. Nikolaev, J. Speth, and B. G. Zakharov, arXiv:hep-ph/ 9708290.

[7] R. J. Holt and C. D. Roberts, Rev. Mod. Phys. 82, 2991 (2010).

[8] B. Z. Kopeliovich, I. K. Potashnikova, B. Povh, and I. Schmidt, Phys. Rev. D 85, 114025 (2012).
[9] J. R. McKenney, N. Sato, W. Melnitchouk, and C. R. Ji, Phys. Rev. D 93, 054011 (2016).

[10] D. Boer et al., arXiv:1108.1713; A. Accardi et al., Eur. Phys. J. A 52, 268 (2016); E. C. Aschenauer, S. Fazio, J. H. Lee, H. Mäntysaari, B.S. Page, B. Schenke, T. Ullrich, R. Venugopalan, and P. Zurita, Rep. Prog. Phys. 82, 024301 (2019).

[11] J. L. A. Fernandez et al. (LHeC Study Group Collaboration), J. Phys. G 39, 075001 (2012); P. Agostini et al., arXiv: 2007.14491.

[12] J. D. Sullivan, Phys. Rev. D 5, 1732 (1972).

[13] U. D'Alesio and H. J. Pirner, Eur. Phys. J. A 7, 109 (2000).

[14] B. Z. Kopeliovich, H. J. Pirner, I. K. Potashnikova, K. Reygers, and I. Schmidt, Phys. Rev. D 91, 054030 (2015).

[15] V. A. Khoze, A. D. Martin, and M. G. Ryskin, Phys. Rev. D 96, 034018 (2017). 
[16] A. B. Kaidalov, V. A. Khoze, A. D. Martin, and M. G. Ryskin, Eur. Phys. J. C 47, 385 (2006).

[17] V. A. Khoze, A. D. Martin, and M. G. Ryskin, Eur. Phys. J. C 48, 797 (2006).

[18] E. Levin and S. Tapia, Nucl. Phys. A892, 1 (2012).

[19] F. Carvalho, V. P. Goncalves, D. Spiering, and F. S. Navarra, Phys. Lett. B 752, 76 (2016).

[20] V. P. Goncalves, D. Spiering, and F. S. Navarra, Phys. Rev. D 93, 054025 (2016).

[21] N. N. Nikolaev and B. G. Zakharov, Z. Phys. C 64, 631 (1994).

[22] F. Gelis, E. Iancu, J. Jalilian-Marian, and R. Venugopalan, Annu. Rev. Nucl. Part. Sci. 60, 463 (2010); H. Weigert, Prog. Part. Nucl. Phys. 55, 461 (2005); J. Jalilian-Marian and Y. V. Kovchegov, Prog. Part. Nucl. Phys. 56, 104 (2006).
[23] R. J. Glauber, in Lecture in Theoretical Physics, edited by W. E. Brittin and L. G. Duham (Interscience, New York, 1959), Vol. 1.

[24] E. Iancu, K. Itakura, and S. Munier, Phys. Lett. B 590, 199 (2004).

[25] G. Soyez, Phys. Lett. B 655, 32 (2007).

[26] J. Olsson (H1 Collaboration), Proc. Sci., DIS2014 (2014) 156; V. Andreev et al. (H1 Collaboration), Eur. Phys. J. C 74, 2915 (2014); F. D. Aaron et al. (H1 Collaboration), Eur. Phys. J. C 68, 381 (2010).

[27] V.P. Goncalves, B. D. Moreira, D. Spiering, and F. S. Navarra, Phys. Rev. D 94, 014009 (2016).

[28] F. Carvalho, V. P. Goncalves, D. Spiering, and F. S. Navarra, Phys. Rev. D 97, 074002 (2018). 